\title{
Low-concentration copper solution jeopardizes larval movement and ability to survive predation: New insight into malaria eradication via vector control
}

\author{
Mohamad Reza, Daisuke S. Yamamoto and Hiroyuki Matsuoka* \\ * Corresponding author: Division of Medical Zoology, Department of Infection and Immunity, Jichi Medical University, \\ 1311-1 Yakushiji, Shimotsuke, Tochigi-ken, 329-0398 Japan (e-mail: hiroyuki@jichi.ac.jp)
}

(Received: 9 May 2012; Accepted: 24 July 2012)

\begin{abstract}
A new approach to eradicating malaria is urgently required. Emphasizing vector eradication via larval control, we tried to find a safe concentration of copper, which can be utilized to reduce larval survival. It has been observed that mosquito larvae died when exposed to solid copper in water. We confirmed this phenomenon in our own experiments. We next studied the minimum copper concentration for effectively killing or disturbing Anopheles stephensi larvae. A concentration of $0.6 \mathrm{ppm}$ had a significant impact on larval movement, making them vulnerable to predatory fish. Feeding time tests showed that guppies (Poecilia reticulata) ate the weakened larvae significantly faster than control larvae. Utilizing low concentrations of copper together with predatory fish in the field may shed new light on malaria eradication via vector control.
\end{abstract}

Key words: Anopheles stephensi, copper, fish, guppy, malaria control, mosquito larva, Poecilia reticulata

\section{INTRODUCTION}

Malaria is still a major problem worldwide, especially in undeveloped and developing countries. There are three common approaches to its control: medical treatment, vaccination, and vector control. The first approach focuses on finding medicines to treat malarial infections. This method however cannot diminish infection rates since malaria parasites have built up resistance to many anti-malarial drugs. Recent study shows that artemisinin-resistant malaria is emerging (Phyo et al. 2012). As the current first treatment involves artemisinin, this means that artemisinin is no longer guaranteed to successfully suppress malaria parasites. Vaccination is not very successful ether, because only one vaccine candidate has been found effective enough to induce immunity, i.e. the RTS, S/AS vaccine (Agnandji et al., 2011). These problems encouraged us to concentrate on vector control, protecting against adult mos- quitoes with insecticides or the usage of mosquito bed-nets (Killeen et al., 2002).

The use of DDT to suppress adult mosquitos is believed to be the ultimate method of eradicating malaria. This approach was preferred in the past because DDT moderately reduces adult longevity and can radically suppress community-level transmission. However, larval control has a less dramatic effect and is more difficult to apply in the field. The most effective campaign against malaria vectors, in Brazil in the 1930s, relied overwhelmingly upon larval control, though many mosquitos have built up resistance to DDT (Hinzoumbe et al., 2008). Although mosquito nets can effectively protect against adult mosquitos at sleeping time, without reducing numbers of the vector itself, mosquitos do not bite only in the sleeping period.

Consequently, we decided to focus on larval control. The larval stage is the most vulnerable stage of the mosquito life cycle. Adult mosquitoes can move freely and so are more 
difficult to control. Larvae, on the other hand, stay in the same environment. Thus, larval stage is an easier target for eradication. Approaches tried in the past include the utilization of fish, oil, and Paris Green (copper acetoarsenite). Many species of fish have proved effective in controlling mosquito larvae (Chandra et al., 2008). The problem is that some larvae survive by hiding at the bottom. The use of oil has the advantage of killing both larvae and pupae, but it can also hinder the growth of vegetation and is not suitable for drinking water. Fish may also be killed when oil is used, or the wind might break up the oil film. Paris Green is a potent larvicide, but its toxicity has become apparent.

Copper, on the other hand, is widely used in daily life. Compared with Paris Green, copper has a safer toxicity range in vertebrate, especially in humans. Despite of several concerns about copper toxicity related to degenerative disease like Alzheimer (Brewer, 2012), which is still in hypothesis level, copper deficiency is actually a more realistic problem lately. Based on the U.S. Environmental Protection Agency's recommendation, 1ppm of copper is safe for drinking water. A recent study (De Romana et al., 2011) introduced the risks and benefits of copper and suggested that the limit of $1 \mathrm{ppm}$ in drinking water should be raised due to copper-deficiency problems. Some studies have revealed that copper has larvicidal capabilities. The peritrophic matrix of aquatic arthropods was perturbed by copper (Beaty et al., 2002). Among eggs of Aedes aegypti (Linnaeus, 1762), $100 \%$ failed to hatch when exposed to 32 ppm of copper, and an $\mathrm{LD}_{50}$ of larvae in 24 hours was achieved with 33 ppm (Keller et al., 1998). However, this concentration is still far above the recommended value for humans $(1 \mathrm{ppm})$. In this study, we try to find the minimum concentration, preferably less than 1 ppm, which can kill mosquito larvae, or at least reduce their ability to survive. By combining this with fish that prey on mosquito larvae, we seek to find a simple and inexpensive method of eradicating malaria via vector control.

\section{Materials And Methods}

\section{Mosquitoes and mice}

Anopheles stephensi Liston, 1901 (strain SDA 500) was reared in our laboratory under room temperature $\left(26^{\circ} \mathrm{C}\right), 50-70 \%$ relative humidity, and a $13: 11 \mathrm{~h}$ of light : dark cycle. From the day of emergence, the mosquitos were provided with a $5 \%$ fructose solution soaked in filter paper. Females five to 20 days after emergence were allowed to feed on anesthetized mice. Three days later, an ovipositing dish was placed in the cage containing gravid females. The eggs were stranded on filter paper soaking in the dish. The filter paper with eggs was placed on a $12 \times 20 \mathrm{~cm}$ hatching tray containing $500 \mathrm{ml}$ of water. After hatching out, 3-5 mg of carp's food per tray was sprinkled on the surface of the water twice daily. Twelve to 20 days later, pupae were collected daily and transferred to cages for adult emergence.

Female BALB/c mice were purchased from SLC (Shizuoka, Japan). The mice were fed ad libitum and exposed to a $13: 11 \mathrm{~h}$ of light: dark cycle. This mosquito-mouse cycle was used to maintain the mosquitoes in our laboratory.

\section{Preparation of copper solutions}

Two copper solutions were used. Copper wire $(1 \mathrm{~g} / 30 \mathrm{~cm})$ was used for the first experiment, which had four conditions: $1.0 \mathrm{~g}$ $(30 \mathrm{~cm}$ of wire), $0.5 \mathrm{~g}(15 \mathrm{~cm})$, or $0.25 \mathrm{~g}$ $(7.5 \mathrm{~cm})$ in $15 \mathrm{ml}$ of water, and $15 \mathrm{ml}$ of water without copper as a control. The water was changed every two days. $\mathrm{CuSO}_{4}$ solutions were used for the second experiment. Copper concentrations were $2.4 \mathrm{ppm}, 1.2 \mathrm{ppm}, 0.6$ $\mathrm{ppm}$ and $0 \mathrm{ppm}$. Each concentration was allocated to a $15 \mathrm{ml}$ container. The $\mathrm{CuSO}_{4}$ solutions were replenished with fresh solution every two days.

\section{Preparation of larvae}

For each concentration (control, the lowest concentration, the medium concentration, and the highest concentration), we prepared three containers. We placed 20 larvae of stage II or III in each container. Thus, 60 larvae 


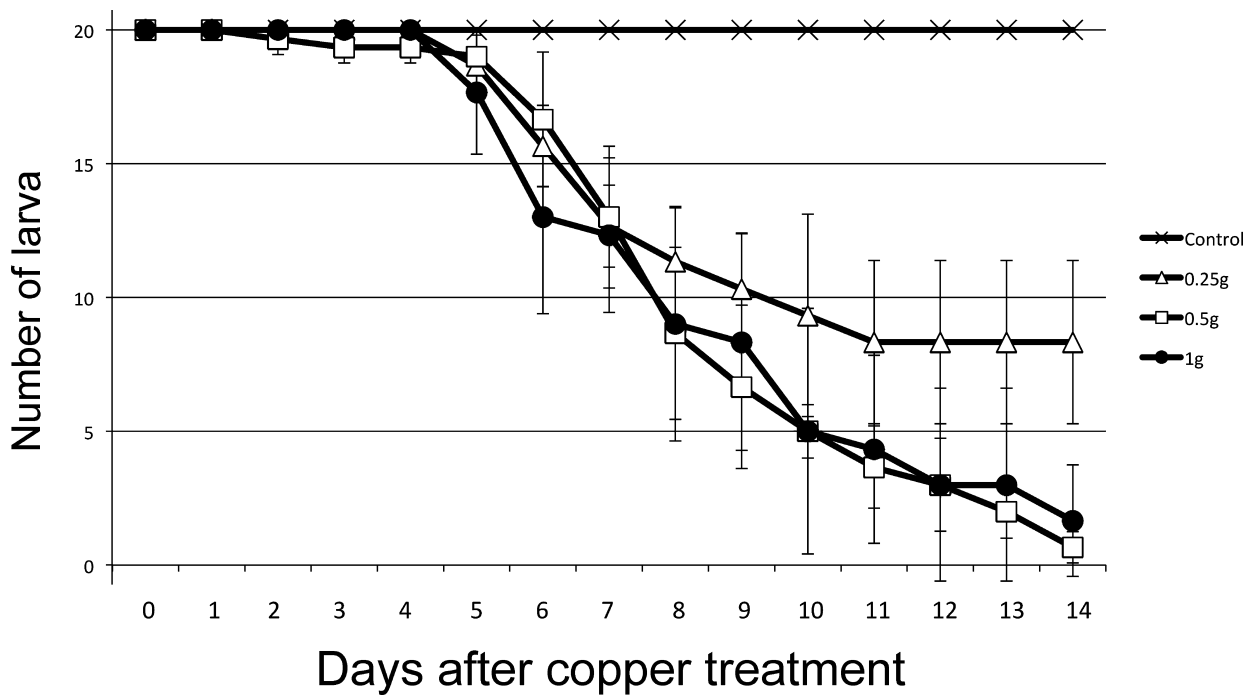

Fig. 1. Copper treatment of An. stephensi larvae and numbers of living larvae. There were four conditions: no copper wire in $15 \mathrm{ml}$ of water, the lowest concentration $(0.25 \mathrm{~g} / 15 \mathrm{ml})$, the medium concentration $(0.5 \mathrm{~g} / 15 \mathrm{ml})$ and the highest concentration $(1 \mathrm{~g} / 15 \mathrm{ml})$. Twenty larvae were placed in each container at Day 0 . Three containers were prepared for each treatment. Vertical axis represent number of larvae. Horizontal axis represent experimental days (total 14 days)

were used for each concentration. Larvae were fed two times a day and the water was changed every two days to keep it clean. We examined the mortality, behavior, and movement of the larvae. Dead larvae were removed from the containers. Emerging pupae were also removed but counted as surviving larvae. Larvae were observed for 14 days, and all parameters were recorded and statistically analyzed.

\section{Fish}

Female guppy fish (Poecilia reticulate Peters, 1859) were utilized to evaluate the ability of the larvae to move. Fifty larvae were dropped in a fish tank containing five starved Guppy. The fish were maintained with anopheline larvae. One fish ate 100 stage II or III larvae every day. The water of the tank was changed twice a week.

\section{Results}

Figure 1 shows that the highest concentration of solid copper $(1 \mathrm{~g} / 15 \mathrm{ml})$ caused significant larval mortality. Mortality started on day 5 and continued until the end of the experiment (day 14). A $50 \%$ lethal dose $\left(\mathrm{LD}_{50}\right)$ was achieved on day 8 , and the remaining larvae failed to become pupae. The next concentration of solid copper $(0.5 \mathrm{~g} / 15 \mathrm{ml})$ also caused a significant mortality, starting from day 2 and persisting until day 14. Although the mortality occurred sharply in the beginning, there was no significant difference with the highest concentration $(1 \mathrm{~g} / 15 \mathrm{ml})$. The lowest concentration of solid copper $(0.25 \mathrm{~g} / 15 \mathrm{ml})$ also caused significant mortality. The mortality started from day 5 and continued until day 10. After day 10, no mortality occurred in this group, perhaps due to adaptation of the larvae or greater resistance of the late instar larvae to a toxic environment. The movement of larvae in the coppertreated group was reduced significantly compared to the control. All larvae in the coppertreated group were unhealthy, as indicated by a smaller size and darkened body color. These phenomena were dose dependent. At the higher dose, development was arrested and the larvae appeared unhealthy. Copper seemed to jeopardize survival, due to a lack of movement and unhealthy state.

We also tried to compare the copper solutions $\left(\mathrm{CuSO}_{4}\right)$ for the capability to kill $A n$. 


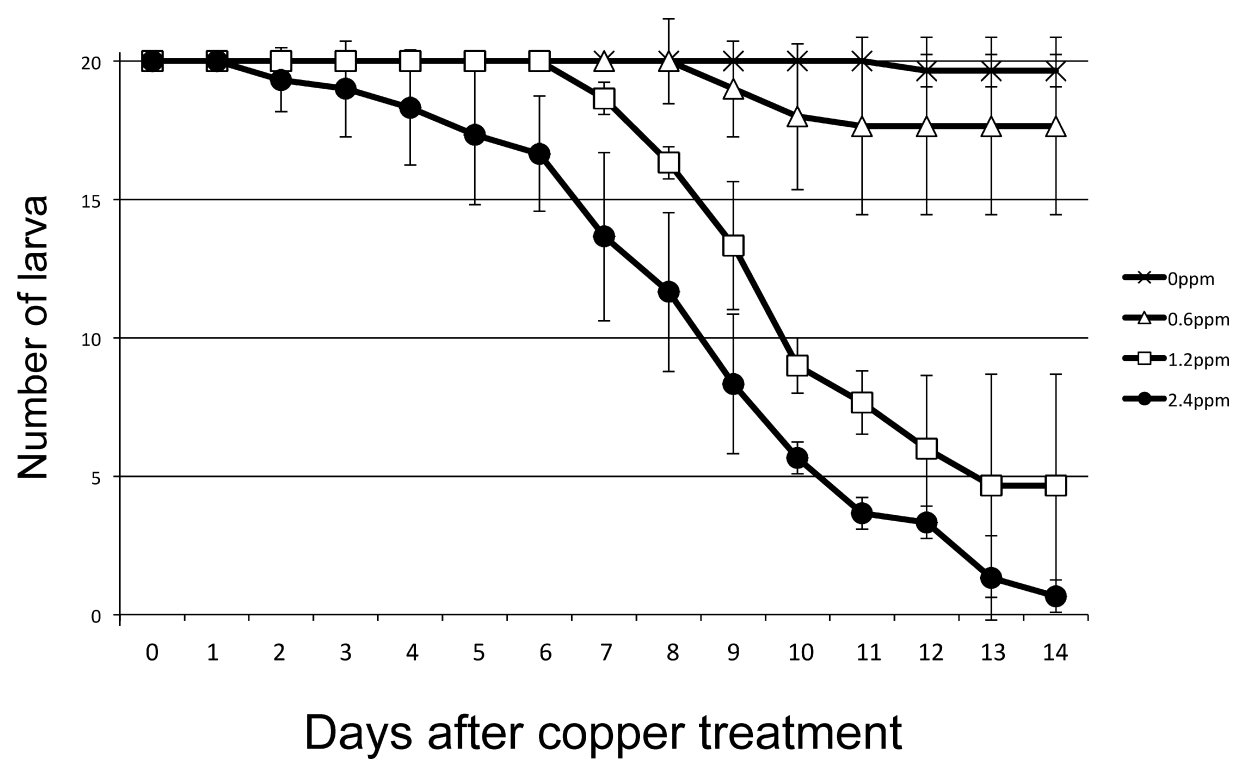

Fig. 2. $\mathrm{CuSO}_{4}$ treatment of An. stephensi larvae and numbers of living larvae. There were four conditions: no copper in $15 \mathrm{ml}$ of water, the lowest concentration ( $0.6 \mathrm{ppm}$ of copper), the medium concentration (1.2 ppm of copper) and the highest concentration (2.4 ppm of copper). Twenty larvae were placed in each container at Day 0 . Three containers were prepared for each treatment. Vertical axis represent number of larvae. Horizontal axis represent experimental days (total 14 days)

stephensi larvae. Figure 2 shows that the highest concentration of copper $(2.4 \mathrm{ppm})$ caused significant mortality. The mortality first occurred on day 2 and continued sharply until the end of the experiment. The $\mathrm{LD}_{50}$ was achieved on day 9 and no pupae appeared until the end of the experiment. The medium concentration (1.2 ppm) also caused significant mortality. The mortality occurred on day 7 and continued until day 12. Mortality no longer occurred after day 12, may be due to the ability to resist a toxic environment or higher tolerance to copper in the late instars. Some pupae successfully emerged from this group from day 7 . The lowest concentration of copper $(0.6 \mathrm{ppm})$ did not produce significant mortality, but caused a lack of movement and developmental arrest like the solid copper.

Next we tried to test the lowest coppertreated group's survival ability by measuring larval feeding times of the guppy ( $P$. reticulata), a well-known predator of larvae. Compared to the control group, the copper-treated larvae $(0.6 \mathrm{ppm})$ were more susceptible to the predation because they were easier to capture
(Figure 3). The guppies took longer time to capture the larvae of the control group because of their survival abilities (movement, diving to the bottom and sense of danger) were still in good condition. Upon encountering predators, healthy larvae acted by evading, moving away or diving to the bottom, which made it more difficult for the predators to catch the larvae. Copper-treated larvae seemed to lose these abilities, which made them easier to catch.

\section{Discussion}

We utilize solid copper (copper wire) and copper solution $\left(\mathrm{CuSO}_{4}\right)$ to determine an effective dose for killing An. stephensi larvae. For copper wire, the effective lethal dose was the lowest mass $(0.25 \mathrm{~g} / 15 \mathrm{ml})$ at day 10 . $\mathrm{CuSO}_{4}$ showed an effective lethal dose at $1.2 \mathrm{ppm}$, the medium concentration. The $\mathrm{LD}_{50}$ was achieved at day 10 . The lowest concentration of copper solution $(0.6 \mathrm{ppm}) \mathrm{did}$ not cause adequate mortality, but resulted in clear morbidity and a reduction in survivability compared to the control. It was very clear that the survival instincts and capabili- 


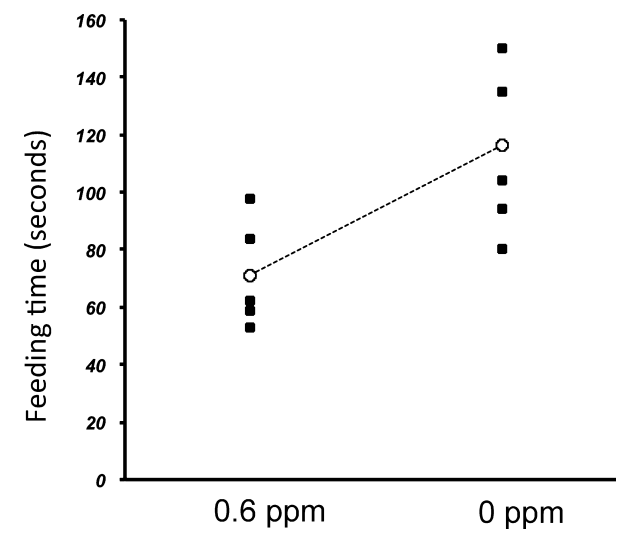

Fig. 3. Feeding times (seconds) for guppies (Poecilia reticulata). Group A: $\mathrm{CuSO}_{4}$-treated (0.6 ppm of copper) larvae. Group B: control (0 ppm) larvae. After 48 hours of exposure to copper, comparative time tests were conducted five times using the same fish. Copper-treated larvae were eaten faster. Two-tail t-tests showed a significant difference $(\mathrm{P}<0.05)$. Vertical axis represent the time of capture (seconds). Solid black-square represent each trial in each group. Empty white circle represent mean point from each group.

ties of the larvae were greatly affected by copper at the lowest concentration (less than 1 $\mathrm{ppm}$ ). This loss led to greater susceptibility to predation by fish.

We further confirmed that the morbidity and less of survival ability made the larvae more vulnerable to attack. The feeding time of the guppies (P. reticulata) was significantly shorter with the copper-treated larvae (0.6 ppm) than the control. The healthy larvae noticed the predators and hid at the bottom, but the copper-exposed larvae could not evade predation. In the laboratory condition the guppies finally captured all larvae in all groups, but we believed that this happened due to the very narrow and small environment of the trays. We expect that in natural large breeding place, where many vegetation and natural hiding place available for the larvae, the predatory fish will fail to capture most of the larvae due to their survival ability. It is also proved that Anopheles sp. can dive to the bottom for a long time (Silver, 2008), which reduces the chance for predatory fish to eliminate Anopheles larvae in their natural habitat.
We believe that by utilizing copper at low concentrations, we can reduce malarial transmission through vector control, since the U.S. Environmental Protection Agency has allowed copper at concentrations of $1 \mathrm{pm}$ and below to be consumed in drinking water. This is the first study to find an exact concentration of copper and examine morbidity and survival among mosquito larvae. There are reports that larvae of some species of mosquito build tolerance to copper, but the concentration of copper was low (around $0.1 \mathrm{ppm}$ ) (Mireji et al., 2008). Therefore, we believe that the combination of low copper treatment of larval habitats and the utilization of indigenous predators such as medaka (Oryzias latipes Temminck \& Schlegel, 1846) will help to eradicate malaria. The bioaccumulation and toxicity of copper in outdoor freshwater environment has also been examined (Hoang et al., 2011). The results show no significant accumulation of copper in fish after treatment. This encourages us to combine a low concentration copper treatment with fish that eat mosquito larvae.

These methods are inexpensive and simple, particularly against malarial vectors in paddy fields. However, further investigation in the field will be required to ensure their success.

\section{ACKNOWLEDGEMENTS}

We thank Ms. Michiyo Soutome, Ms. Chisato Seki, Ms. Keiko Watano and Mr. Jin Sato for handling of the mosquitoes, mice and fish. This work was supported by a grant of the Support Program for Strategic Research Platform for Private Universities from the Japanese Ministry of Education, Culture, Sport, Science and Technology to HM. MR was financially supported as a $\mathrm{PhD}$ student from the Indonesian Government.

\section{CONFlict of InTERest}

The authors have no conflict of interest.

\section{REFERENCES}

Agnandji, S. T., Lell, B., Soulanoudjingar, S. S., Fernandes, J. F., Abossolo, B. P. et al. 2011. First results of phase 3 trials of RTS, S/AS01 malaria vaccine in African children. N. Engl. J. Med., 365: 1863-1875. 
Beaty, B. J., Mackie, R. S., Mattingly, K. S., Carlson, J. O. and Keller, A. R. 2002. The midgut epithelium of aquatic arthropods: a critical target organ in environmental toxicology. Environ. Health Perspect, 110: 911914.

Brewer, G. J. 2012. Copper toxicity in Alzheimer's disease: cognitive loss from ingestion of organic copper. J. Trace Elem. Med. Biol., 26: 89-92.

Chandra, G., Bhattacharjee, I., Chatterjee, S. N. and Ghosh, A. 2008. Mosquito control by larvivorous fish. Indian J. Med. Res., 127: 13-27.

De Romana, D. L., Olivares, M., Uauy, R., Araya, M. 2011. Risk and benefits of copper in light of new insight of copper homeostasis. J. Trace Elem. Med. Biol., 25: 3-13.

Hinzoumbe, C. K., Peka, M., Nwane, P., Gouni, I. D., Etang, J., Ekobo, A. S. and Simard, F. 2008. Insecticide resistance in Anopheles gambiae from south-western Chad, Central Africa. Malaria J., 7: 192.

Hoang, T. C., Pryor, R. L., Rand, G. M. and Frakes, R. A. 2011. Bioaccumulation and toxicity of copper in outdoor freshwater microcosm. Ecotoxicol. Environ. Saf., 74: 1011-1020.
Keller, A. R., Olson, K. E., McGaw, M., Oray, C., Carlson, J. O. and Beaty, B. J. 1998. Effect of heavy metal on Aedes aegypti (Diptera : culicidae) larvae. Ecotoxicol. Environ. Saf., 39: 41-47.

Killeen, G. F., Fillinger, U., Kiche, I., Gouagna, C. L. and Knols, B. G. J. 2002. Eradication of Anopheles gambiae from Brazil: lessons for Malaria control in Africa? Lancet Infect. Dis., 2: 618-627.

Mireji, P. O., Keating, J., Hassanali, A., Mbogo, C. M., Nyambaka, H., Kahindi, S. and Beier, J. C. 2008. Heavy metals in mosquito larval habitats in urban Kisumu and Malindi, Kenya and their impact. Ecotoxicol. Environ. Saf., 70: 147-153.

Phyo, A. P., Nkhoma, S., Stepniewska, K., Ashley, E. A., Nair, S., McGready, R., Moo, C. L., Al-Saii, S., Dondorp, A. M., Lwin, K. M., Singhasivanon, P., Day, N. P. J., White, N. J., Anderson, T. J. C. and Nosten, F. 2012. Emergence of artemisinin-resistant malaria on the western border of Thailand: a longitudinal study. Lancet, 379: 1960-1966.

Silver, J. B. 2008. Mosquito ecology, Field sampling methods, third edition, 1494 pp., Springer, Netherlands. 